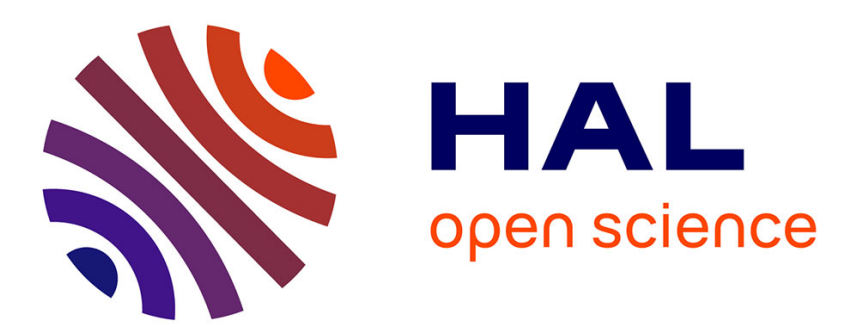

\title{
Windfield estimation by radar track Kalman filtering and vector spline extrapolation
}

\author{
Daniel Delahaye, Stéphane Puechmorel, Pierre Vacher
}

\section{To cite this version:}

Daniel Delahaye, Stéphane Puechmorel, Pierre Vacher. Windfield estimation by radar track Kalman filtering and vector spline extrapolation. DASC 2003, 22nd Digital Avionic Systems Conference, Oct 2003, Indianapolis, United States. pp 5.E.2 - 51-11 vol.1, 10.1109/DASC.2003.1245869 . hal00938037

\section{HAL Id: hal-00938037 \\ https://hal-enac.archives-ouvertes.fr/hal-00938037}

Submitted on 5 May 2014

HAL is a multi-disciplinary open access archive for the deposit and dissemination of scientific research documents, whether they are published or not. The documents may come from teaching and research institutions in France or abroad, or from public or private research centers.
L'archive ouverte pluridisciplinaire $\mathbf{H A L}$, est destinée au dépôt et à la diffusion de documents scientifiques de niveau recherche, publiés ou non, émanant des établissements d'enseignement et de recherche français ou étrangers, des laboratoires publics ou privés. 


\title{
WINDFIELD ESTIMATION BY RADAR TRACK KALMAN FILTERING AND VECTOR SPLINE EXTRAPOLATION
}

\author{
Daniel Delahaye CENA 7, Ave Ed Belin 31055 Toulouse France (delahaye@tls.cena.fr) \\ Stephane Puechmorel ENAC 7, Ave Ed Belin 31055 Toulouse France (puechmor@recherche.enac.fr) \\ Pierre Vacher ONERA-CERT 2, Ave Ed Belin 31055 Toulouse France (pierre.vacher@cert.fr)
}

\begin{abstract}
Accurate wind magnitude and direction estimation is essential for aircraft trajectory prediction. For instance, based on these data, one may compute entry and exit time from a sector or detect potential conflict between aircraft. Since the flight path has to be computed and updated on real time for such applications, wind information has to be available in real time too. The wind data which are currently available through meteorological service broadcast suffer from small measurement rate with respect to location and time. In this paper, a new wind estimation method based on radar tracks is developed. An Extended Kalman filter extracts the wind information by observation of the radar tracks in turns. After performing many evaluations in realistic frameworks, our approach is able to estimate the wind vectors accurately. By this mean, each aircraft can be seen as a wind sensor when it is turning. Based on those measurements, a global space-time wind field estimation using vector splines is extrapolated in order to produce wind maps in the area of interest. The underline model for wind field computation is Shallow-Water, which assumes geostrophic wind. The accuracy of this wind map is dependent of the number of aircraft turns in a given zone; then the estimation is better in the terminal area (TMA) than in en-route area because aircraft are tuning more often. Further improvements to the estimation can be made by correlating with meteorological measurements.
\end{abstract}

\section{Introduction}

When an aircraft flies from a city A to a city B, it has to be managed by air traffic controllers in order to avoid collisions with others aircraft. Everyday, about 8000 aircraft fly in the French airspace, inducing a huge amount of control workload. Such a workload, is then spread by the mean of the airspace sectoring. The airspace is divided into geometrical sectors, each of them being assigned to a controller team. When a conflict between two (or more) aircraft is detected, the controller changes their routes (heading, speed, altitude) in order to keep a minimum distance between them during the crossing. All flying aircraft are then monitored during their navigation and so from the departure till the destination. The controller has a $2 \mathrm{D}$ visualization of his traffic by the mean of the radar screen. At the beginning of radar air traffic control, civil aviation authority used primary radar. In such a system, the radar send a big electromagnetic impulsion (several mega watt (peak power) during one micro second) in the airspace waiting the echo back. This impulsion propagates at the light speed ( $c=300 \mathrm{~km}$ per second), touch the aircraft and comes back to the radar with a very low power (some nano watts). Based on the time difference between the two impulsions, it is easy to compute the distance between the radar and the aircraft $\left(\right.$ diff $\left._{\text {distance }}=\frac{{ }_{\text {diffine. }}}{2}\right)$. This system gives a straight measure of the distance between the aircraft and the radar which is a mix between the 2D geographical distance and the altitude of the aircraft. When controllers used only primary radar, they had to asked the pilot to give his altitude and his ID in order to identify more precisely the spot they saw on their screen. The secondary radar really improved this system by the mean of active target concept. In this system, the aircraft is not passive anymore (from the radar point of view) but is equipped by an emitter which answer to the radar when the radar beam touch the aircraft. Every time the aircraft is addressed by the radar, the aircraft emitter send back 
two codes called ModeA and ModeC. Those numbers are coded into octal base and give respectively the $\mathrm{ID}$ and the altitude of the aircraft. When the radar receives back those two pieces of information, it is easy to compute the $2 \mathrm{D}$ geographical distance and the altitude of the aircraft. Another radar system, called Mode S, proposes to establish a data link between the radar and the aircraft during the time the aircraft is in the radar beams. This short connection enables to downlink some flight data to the ground such like roll angle, true air speed, heading, altitude rate, etc.....and enable to improve the radar tracking [1].

This communication between aircraft and the ground can be extended by the mean of satellites which are even accessible over the oceans where there is no radar. All those radars deliver raw measures with additional noises coming from electromagnetic perturbations, electronic systems, etc.... Those measures are then filtered by the mean of a tracker filter in order to produce smooth trajectories.

When a controller observes its traffic on the radar screen, he tries to identify convergent aircraft which may be in conflict in a near future, in order to apply maneuvers that will separate them. The problem is to estimate where the aircraft will be located in this near future (5-10 minutes); this process is call trajectory prediction. This prediction may be also very useful in order to estimate the workload level in control sector to prevent over capacity event. As a mater of fact, it is very useful to estimate when an aircraft will enter a sector in order to compute the associated sector workload and to apply regulation if necessary. When a sector is expected to be overloaded, the aircraft involved in such a process will be speeded up or slow down by the controller in order to adapt the demand to the actual capacity as much as possible. The trajectory prediction depends mainly on the residual noise after filtering (see [2]), the weight of the aircraft, the temperature and the wind. The residual noise is integrated with time with a growing covariance matrice indicating that the estimated position is less and less accurate. The weight of the aircraft is relevant in the flight dynamic model but is still a raw data. The engines of aircraft are sensitive to the air temperature and such a data is very useful to model the trust of the aircraft but it is also very difficult to measure on real time. Finally, the wind influences strongly the cinematic of the aircraft and limits also the trajectory prediction. Based on the available accuracy, the actual limit of the trajectory prediction is about 15 minutes. It means that after 15 minutes; the uncertainty is so big that the estimated position is no more useful for any ATM applications. Several efforts have tried to improved the trajectory prediction by estimating or suppressing the wind [3], [4], [5], [6], [7]. The present paper, proposes a new method for estimating the wind around aircraft by the mean of observations of the radar tracks and some downlink data. One goal of this work is to show how it is possible to extract wind information from the radar observations.

The paper is organized as follow : the first part present the Kalman filter and its non linear extension called Extended Kalman Filter. The second part describes two models adapted for the wind estimation. The third part presents some results and compare the performance of our two models. Finally, the fourth part describes how a wind field map can be built using some spread measures of the wind.

\section{Kalman Filtering}

\section{Linear Form}

The Kalman filter is named after Rudolph E.Kalman who in 1960 published his famous paper describing a recursive solution to the discrete-data linear filtering problem [8]. The Kalman filter is essentially a set of mathematical equations that implement a predictor-corrector type estimator that is optimal in the sense that it minimizes the estimated error covariance (when some presumed conditions are met). It has been successfully applied in many real problems [9], [10], 11 ], [12], [13]. The following description of the Kalman filter is mainly due to G.Welch and G.Bishop [14].

The Kalman filter addresses the general problern of trying to estimate the state $\underline{X}(k) \in \mathbb{R}^{n}$ of a discrete controlled process that is governed by the linear stochastic difference equation call evolution equation :

$$
\underline{X}(k+1)=\underline{F}(k) \cdot \underline{X}(k)+\underline{G}(k) \cdot \underline{\hat{U}}(k)+\underline{v}(k)
$$


with a measurement $\underline{Z}(k) \in \mathcal{R}^{m}$ :

$$
\underline{Z}(k)=\underline{H}(k) \cdot \underline{X}(k)+\underline{w}(k)
$$

The $n . n$ matrix $F(k)$ in the evolution equation 1 relates the state at the present step $k$ to the state at the next step $k+1$, in the absence of either a driving function or process noise. The n.p matrix $G(k)$ relates the optional control input $\underline{\underline{U}}(k) \in \mathcal{R}^{p}$ to the state $\underline{X}$. The $n . m$ matrix $\underline{H}(k)$ in the measurement equation 2 relates the state to the measurement $\underline{Z}(k)$. The random variables $\underline{v}(k)$ and $\underline{w}(k)$ represent the process and measurement noise (respectively). They are assumed to be independent (of each other), centered, white, and with normal probability distributions. Furthermore the optional control is disturbed by a random variable $\underline{n}(k)$ $(\underline{\hat{U}}(k)=\underline{U}(k)+\underline{n}(k))$ which will be supposed to have a normal probability distribution also.

$$
\left\{\begin{array}{lll}
p(\underline{v}(k)) & \sim \mathcal{N}(0, \underline{Q}(k)) \\
p(\underline{w}(k)) & \sim \mathcal{N}(0, \underline{R}(k)) \\
p(\underline{n}(k)) & \sim \mathcal{N}(0, \underline{N}(k))
\end{array}\right.
$$

So we have :

$$
\begin{array}{ll}
E[\underline{\underline{v}}(k)]=0 & E\left[\underline{v}(k) \underline{v}(j)^{T}\right]=\underline{Q}(k) \cdot \delta_{k j} \\
E[\underline{w}(k)]=0 & E\left[\underline{w}(k) \underline{w}(j)^{T}\right]=\underline{R}(k) \cdot \delta_{k j} \\
E[\underline{\underline{n}}(k)]=0 & E\left[\underline{\underline{n}}(k) \underline{\underline{n}}(j)^{T}\right]=\underline{N}(k) \cdot \delta_{k j}
\end{array}
$$

where ${ }^{T}$ is the transpose operator and $\delta_{k j}$ is the Kronecker symbol which is 1 if $k=j$ and 0 if $k \neq j$. We define $\underline{\hat{X}}(k+1 / k) \in R^{n}$ to be the a priori state estimate at step $k+1$ given knowledge of the process prior to step $k+1$, and $\underline{\hat{X}}(k+1 / k+1) \in R^{n}$ to be the a posteriori state estimate at step $k+1$ given measurement $\underline{Z}(k+1)$.

We can then define a priori and a posteriori estimate error as :

$$
\begin{aligned}
& \underline{e}(k+1 / k)=\underline{X}(k+1)-\underline{\hat{X}}(k+1 / k) \\
& \underline{e}(k+1 / k+1)=\underline{X}(k+1)-\underline{\hat{X}}(k+1 / k+1)
\end{aligned}
$$

The a priori estimate error covariance is then:

$$
\underline{P}(k+1 / k)=E\left[\underline{e}(k+1 / k) \underline{e}(k+1 / k)^{T}\right]
$$

and the a posteriori estimate error covariance is:

$$
\begin{gathered}
\underline{P}(k+1 / k+1)= \\
E\left[\underline{e}(k+1 / k+1) \underline{e}(k+1 / k+1)^{T}\right]
\end{gathered}
$$

In deriving the equation for the Kalman filter, we begin with the goal of finding an equation that computes an a posteriori state estimate $\underline{\hat{X}}(k+1 / k+1)$ as a linear combination of an a priori estimate $\underline{\hat{X}}(k+1 / k)$ and a weighted difference between an actual measurement $\underline{Z}(k+1)$ and a measurement prediction $\underline{H} \cdot \underline{\underline{X}}(k+1 / k)$ :

$$
\begin{gathered}
\underline{\hat{X}}(k+1 / k+1)=\underline{\hat{X}}(k+1 / k)+ \\
\underline{K}(k+1) \cdot(\underline{Z}(k+1)-\underline{H} \cdot \underline{\hat{X}}(k+1 / k))
\end{gathered}
$$

The difference $(\underline{Z}(k+1)-\underline{H} \cdot \underline{\hat{X}}(k+1 / k))$ in equation 8 is called the measurement innovation, or the residual. The residual reflects the discrepancy between the predicted measurement $\underline{H} \cdot \underline{\underline{X}}(k+1 / k)$ and the actual measurement $\underline{Z}(k+1)$. A residual of zero means that the two are in complete agreement. The $n . m$ matrix $\underline{K}(k+1)$ in equation 8 is chosen to be the gain or blending factor that minimizes the a posteriori error covariance 7 . This minimization can be accomplished by first substituting equation 8 into the above definition for $\underline{e}(k+1 / k+1)$, substituting that into equation 7 , performing the indicated expectations, taking the derivative of the trace of the result with respect to $\underline{K}(k+1)$, setting that result equal to zero, and then solving for $\underline{K}(k+1)$. One form of the resulting $\underline{K}(k+1)$ that minimizes equation 7 is given by :

$$
\begin{gathered}
\underline{K}(k+1)=\underline{P}(k+1 / k) \cdot \underline{H}(k+1)^{T} . \\
{\left[\underline{H}(k+1) \underline{P}(k+1 / k) \underline{H}(k+1)^{T}+\underline{R}(k+1)\right]^{-1}}
\end{gathered}
$$

Looking at equation 9 we see that as the measurement error covariance $\underline{R}(k+1)$ approaches zero, the gain $\underline{K}(k+1)$ weights the residual more heavily. Specifically :

$$
\lim _{\underline{R}(k+1) \rightarrow \underline{0}} \underline{K}(k+1)=\underline{H}^{-1}
$$

On the other hand, as the a priori estimate error covariance $\underline{P}(k+1 / k)$ approaches zero, the gain $\underline{K}(k+1)$ weights the residual less heavily.

Specifically :

$$
\lim _{\underline{P}(k+1 / k) \rightarrow \underline{0}} \underline{K}(k+1)=0
$$

As the measurement error covariance $R(k+1)$, the actual measurement $\underline{Z}(k+1)$ is "trusted" more and more, while the predicted measurement $\underline{H} . \underline{\hat{X}}(k+1 / k)$ is trusted less and less. On the other hand, as the a priori estimate error covariance 
$P(k+1 / k)$ approaches zero the actual measurement $\underline{Z}(k+1)$ is trusted less and less, while the predicted measurement $\underline{H} \cdot \underline{\hat{X}}(k+1 / k)$ is trusted more and more.

The Kalman filter estimates a process by using a form of feedback control : the filter estimates the process state at some time and then obtains feedback in the form of (noisy) measurements. As such, the equation for the Kalman filter fall into two groups : time update equations and measurement update equations. The time update equations are responsible for projecting forward (in time) the current state and error covariance estimates to obtain the a priori estimates for the next time step. The measurement update equations are responsible for the feedback -i.e. for incorporating a new measurement into the a priori estimate to obtain an improved estimate. The time update equations are the following :

$$
\left\{\begin{aligned}
\underline{\hat{X}}(k+1 / k)= & \underline{F}(k) \cdot \underline{X}(k)+\underline{G}(k) \cdot \underline{U}(k) \\
\underline{P}(k+1 / k)= & \underline{F}(k) \cdot \underline{P}(k / k) \cdot \underline{F}(k)^{T}+ \\
& \underline{G}(k) \cdot \underline{N}(k) \cdot \underline{G}(k)^{T}+\underline{Q}(k)
\end{aligned}\right.
$$

The first equation is the state prediction and the second one gives the associated covariance.

The measurement update equations are the following :

$$
\begin{cases}\underline{K}(k+1) & =\underline{P}(k+1 / k) \cdot \underline{H}(k+1)^{T}\left[\underline{H}(k+1) \underline{P}(k+1 / k) \underline{H}(k+1)^{T}+\underline{R}(k+1)\right]^{-1} \\ \underline{\hat{X}}(k+1 / k+1)=\underline{\hat{X}}(k+1 / k)+\underline{K}(k+1) \cdot(\underline{Z}(k+1)-\underline{H}(k+1) \cdot \underline{\hat{X}}(k+1 / k)) \\ \underline{P}(k+1 / k+1)=\underline{\underline{I}}-\underline{K}(k+1) \cdot \underline{H}(k+1)] \cdot \underline{P}(k+1 / k)\end{cases}
$$

The first task during the measurement update is to compute the Kalman gain, $\underline{K}(k+1)$. The next step is to actually measure the process to obtain $\underline{Z}(k+1)$, and then to generate an a posteriori state estimated by incorporating the measurement. The final step is to obtain an a posteriori error covariance estimate.

After each time and measurement update pair, the process is repeated with the previous a posteriori estimates used to project or predict the new a priori estimate. In order to initiate the process, the first initial state and the associated covariance has to be set up. Usually, the first state is build with an initial measure and the associated covariance is set up with the measure covariance or with a big values, because the filter has no confidence in its initial state (it comes from a measure).

\section{Extended Form}

The standard Kalman filter addresses the general problem of trying to estimate the state $\underline{X}(k) \in R^{n}$ of a discrete-time controlled process that is governed by a linear stochastic difference equation. But what happens if the process to be estimated and (or) the measurement relationship to the process is non-linear? A Kalman filter that linearized about the current mean and covariance is referred to as an extended Kalman filter or EKF. We can linearize the estimation around the current estimate using the partial derivative of the process and measurement functions to compute estimates even in the face of non-linear relationships. Let us assume that our process again has a state vector $\underline{X}(k) \in R^{n}$, but that process is now governed by the non-linear stochastic difference equation :

$$
\underline{X}(k+1)=\underline{\mathcal{F}}[k, \underline{X}(k), \underline{U}(k)]+\underline{v}(k)
$$

with a measurement $\underline{Z}(k) \in R^{m}$ that is :

$$
\underline{Z}(k+1)=\underline{\mathcal{H}}[k, \underline{X}(k)]+\underline{w}(k)
$$

where the random variable $\underline{v}(k)$ and $\underline{w}(k)$ again represent the process and measurement noise. The new time update equations are now the followings :

$$
\left\{\begin{aligned}
\underline{\hat{X}}(k+1 / k)= & \frac{\mathcal{F}}{\underline{P}}(k, \underline{X}(k), \underline{U}(k)] \\
\underline{\underline{\mathcal{F}}}(k+1 / k)= & \underline{\mathcal{F}}_{\underline{\underline{X}}}(k) \cdot \underline{\underline{P}}(k / k) \cdot \underline{\underline{\mathcal{F}}}(k) \cdot \underline{\mathcal{F}}_{\underline{X}}(k)^{T}+\underline{Q}(k)
\end{aligned}\right.
$$

where $\mathcal{F}_{\underline{x}}(k)$ is the Jacobian matrix of partial derivatives of $\underline{F}$ with respect to the vector $\underline{X}$ and is given by the following :

$$
\underline{\mathcal{F}}_{\underline{X}}(k) \hat{=}\left[\nabla \underline{X}\left(\underline{\mathcal{F}}[k, \underline{X}, \underline{U}]^{T}\right)\right]_{\underline{X}=\underline{\hat{X}}(k / k), \underline{U}=\underline{\hat{0}}(k)}^{T}
$$


where $\quad \nabla \underline{\underline{s}} \hat{=}\left[\frac{\partial}{\partial S_{1}}, \frac{\partial}{\partial S_{2}}, \ldots, \frac{\partial}{\partial S_{n} s}\right]$

is the differential operator.

The linearized measurement update is now given by:

$\left\{\begin{array}{l}\underline{K}(k+1)=\underline{P}(k+1 / k) \cdot \underline{\mathcal{H}}_{\underline{X}}(k+1)^{T}\left[\underline{\mathcal{H}} \underline{X}(k+1) \cdot \underline{P}(k+1 / k) \underline{\mathcal{H}}_{\underline{X}}(k+1)^{T}+\underline{R}(k+1)\right]^{-1} \\ \underline{\underline{X}}(k+1 / k+1)=\underline{\hat{X}}(k+1 / k)+\underline{\underline{K}}(k+1) \cdot[\underline{Z}(k+1)-\underline{\mathcal{H}}(k, \underline{\hat{X}}(k+1 / k))] \\ \underline{P}(k+1 / k+1)=\left[\underline{\underline{I}}-\underline{K}(k+1) \cdot \underline{\mathcal{H}}_{\underline{X}}(k+1)\right] \cdot \underline{P}(k+1 / k)\end{array}\right.$

More information about the Kalman filter may be found in [15]. Those forms have been used in our wind estimation problem for which the associated models are now presented.

\section{Models}

\section{Model without Command}

The model used for this application supposes that aircraft are flying at constant speed and are turning with a constant air turning rate $\omega_{a}(k)$. This turning rate is also supposed to be always known without noise. This last hypothesis will enable to use the linear form of the Kalman filter. The wind is also supposed to be constant. This simple model will show that wind can be extracted by observing the radar tracks in the turns.

The state vector is given by :

$$
\underline{X}(k)=\left[x_{k} y_{k} T x_{k} T y_{k} W x_{k} W y_{k}\right]^{T}
$$

where $x_{k}, y_{k}$ is the position, $T x_{k}, T y_{k}$ the True Air Speed (TAS) and $W x_{k}, W y_{k}$ the wind.

The experimentation have been done in 2 dimensions (for having shorter equations) but the extension to the third dimension can be done without problem.

The measure vector consists in the radar position:

$$
\underline{Z}(k)=\left[x_{m}(k) y_{m}(k)\right]^{T}
$$

The structure of the system is the following :

$$
\underline{X}(k+1)=\underline{F}(k) \cdot \underline{X}(k)+\underline{v}(k)
$$

$\underline{R}(k)=E\left[\underline{w}(k) \underline{w}(k)^{T}\right]=\left[\begin{array}{l}\sigma_{\rho}^{2} \cdot \sin ^{2}\left(\theta_{0}\right)+\rho_{0}^{2} \cdot \sigma_{\theta}^{2} \cdot \cos ^{2}\left(\theta_{0}\right) \\ \left(\sigma_{\rho}^{2}-\rho_{0}^{2} \cdot \sigma_{\theta}^{2}\right) \frac{\sin \left(2 \theta_{0}\right)}{2}\end{array}\right.$ where

$$
\begin{gathered}
\underline{F}(k)=\left[\begin{array}{cccccc}
1 & 0 & C_{1}\left(\omega_{a}\right) & C_{2}\left(\omega_{a}\right) & \Delta_{t} & 0 \\
0 & 1 & -C_{2}\left(\omega_{a}\right) & C_{1}\left(\omega_{a}\right) & 0 & \Delta_{t} \\
0 & 0 & C_{3}\left(\omega_{a}\right) & C_{4}\left(\omega_{a}\right) & 0 & 0 \\
0 & 0 & -C_{4}\left(\omega_{a}\right) & C_{3}\left(\omega_{a}\right) & 0 & 0 \\
0 & 0 & 0 & 0 & 1 & 0 \\
0 & 0 & 0 & 0 & 0 & 1
\end{array}\right] \\
\underline{Z}(k)=\left[\begin{array}{cccccc}
1 & 0 & 0 & 0 & 0 & 0 \\
0 & 1 & 0 & 0 & 0 & 0
\end{array}\right] . \underline{X}(k)+\underline{w}(k)
\end{gathered}
$$

where

$$
\left\{\begin{array}{l}
C_{1}\left(\omega_{a}(k)\right)=\frac{\sin \left(\omega_{a}(k) \Delta_{t}\right)}{\omega_{a}(k)} \\
C_{2}\left(\omega_{a}(k)\right)=\frac{1-\cos \left(\omega_{a}(k) \Delta_{t}\right)}{\omega_{a}(k)} \\
C_{3}\left(\omega_{a}(k)\right)=\cos \left(\omega_{a}(k) \Delta_{t}\right) \\
C_{4}\left(\omega_{a}(k)\right)=\sin \left(\omega_{a}(k) \Delta_{t}\right)
\end{array}\right.
$$

$\Delta_{t}$ is the time period between two samples ( $k$ and $k+1$ ). This simple model being exact the matrix $\underline{Q}=\underline{0}$ (if aircraft are changing their speeds, this matrix has to be set up in order to enable the Kalman filter to follow such trajectories (with a poorer estimate of the wind)).

The initial measures produced by the radar are expressed in the polar coordinates $((\rho, \theta))$ and the associated standard deviations are $:\left(\sigma_{\rho}=0.12 \mathrm{NM}\right.$ $\left.\sigma_{\theta}=0.14^{\circ}\right)$ for a secondary radar. The state vector being expressed in the Cartesian coordinate $(x, y)$, the noise covariance matrix $\underline{R}(k)$ is build by projection of polar noises onto the Cartesian coordinate system and is given by : $\left.\begin{array}{l}\left(\sigma_{\rho}^{2}-\rho_{0}^{2} \cdot \sigma_{\theta}^{2}\right) \frac{\sin \left(2 \theta_{0}\right)}{2} \\ \sigma_{\rho}^{2} \cdot \cos ^{2}\left(\theta_{0}\right)+\rho_{0}^{2} \cdot \sigma_{\theta}^{2} \cdot \sin ^{2}\left(\theta_{0}\right)\end{array}\right]$ 
where $\rho_{0}$ is the distance between the aircraft and the radar, $\theta_{0}$ is the azimuth of the aircraft.

This model will extract the wind from the radar observations by using a filtered downlinked air turning rate. When such a turning rate is not filtered, it can be included in the command of the filter in order to build a more realistic filter which is presented now in the following section.

\section{Model with Command}

The state vector is the same as in the previous model :

$$
\underline{X}(k)=\left[x_{k} y_{k} T x_{k} T y_{k} W x_{k} W y_{k}\right]^{T}
$$

The measure vector consists in the radar position and the True Air Speed measures:

$$
\underline{Z}(k)=\left[x_{m}(k) y_{m}(k) T_{x m}(k) T_{y m}(k)\right]^{T}
$$

and the command vector is build with the air turning rate $\omega_{a}(k)$ and the acceleration of the aircraft $\gamma(k)$ :

$$
\underline{U}(k)=\left[\omega_{a}(k) \gamma(k)\right]^{T}
$$

The measure $T_{x m}(k), T_{y m}(k)$ and the command $U(k)$ are supposed to be downlinked from the aircraft.

The model is now non linear and has the following structure :

$$
\begin{aligned}
& \underline{X}(k+1)=\underline{\mathcal{F}}[k, \underline{X}(k), \underline{U}(k)]+\underline{v}(k) \\
& \underline{Z}(k+1)=\underline{\mathcal{H}}[k, \underline{X}(k)]+\underline{w}(k)
\end{aligned}
$$

where

$\underline{\mathcal{F}}=\left[\begin{array}{c}x(k)+\left\{C_{1}+D . C_{5}\right\} T x(k)+\left\{C_{2}+D . C_{6}\right\} T y(k)+W x(k) \\ y(k)-\left\{C_{2}+D . C_{6}\right\} T x(k)+\left\{C_{1}+D . C_{5}\right\} T y(k)+W y(k) \\ (1+D)\left(C_{3} . T x(k)+C_{4} \cdot T y(k)\right) \\ (1+D)\left(-C_{4} \cdot T x(k)+C_{3} \cdot T y(k)\right) \\ W x(k) \\ W y(k)\end{array}\right]$

where $C_{1}, C_{2}, C_{3}, C_{4}$ are the same as in the first model and

$$
\left\{\begin{array}{l}
C_{5}=\Delta_{t} \cdot C_{1}-\frac{C_{2}}{\omega_{a}(k)} \\
C_{6}=\frac{C_{l}}{\omega_{a}(k)}-\Delta_{t} \cdot \frac{C_{3}}{\omega_{a}(k)} \\
D=\frac{\gamma \cdot \Delta_{t}}{\sqrt{T x^{2}(k)+T y^{2}(k)}}
\end{array}\right.
$$

Those equations have been linearized in order to build the extended Kalman filter. The matrix $Q$ has been set up to $\underline{0}$ for the first experiments. When the averaged wind is changing during the flight (which is the real framework), the values related to $W x(k), W y(k): \sigma_{W x}, \sigma_{W y}$ must be adjusted to the max change between to samples (for the high altitude wind this max value is about $0.1 \mathrm{kts}$ ).

\section{Results}

\section{Simulation Framework}

In order to test and compare those filters, a radar simulator has been used to produce the reference trajectories which are disturbed several times (30).
Those disturbed trajectories are then filtered by the Kalman filters which generate the filtered trajectories which are then compared to the reference trajectories. This process enable to compute Monte Carlo statistics for the validation of the filters. This framework is summarized on figure 1 (see the end of the paper). 


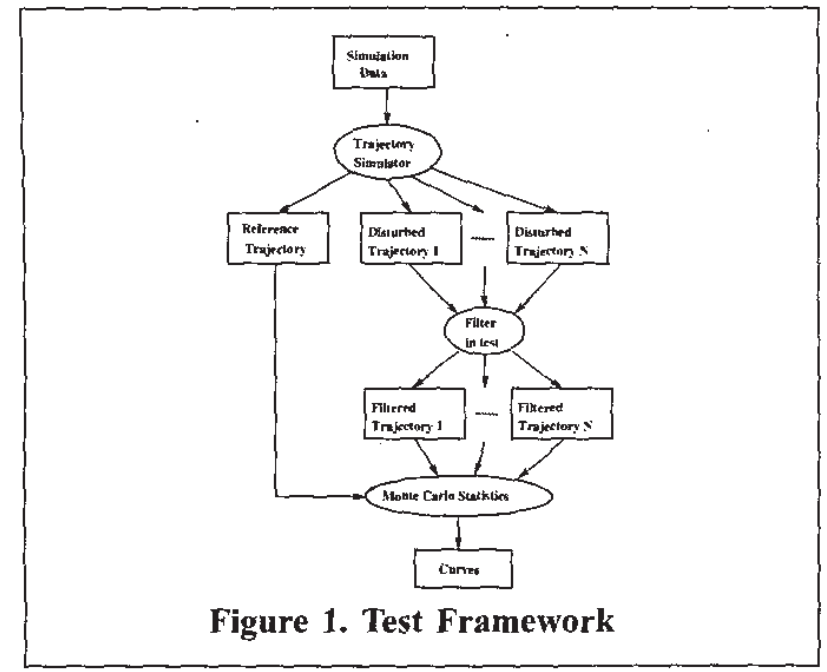

The first trajectory used for our applications is built with 3 straight lines (10 minutes for each) connected with turns as it can be seen on figure 2 .

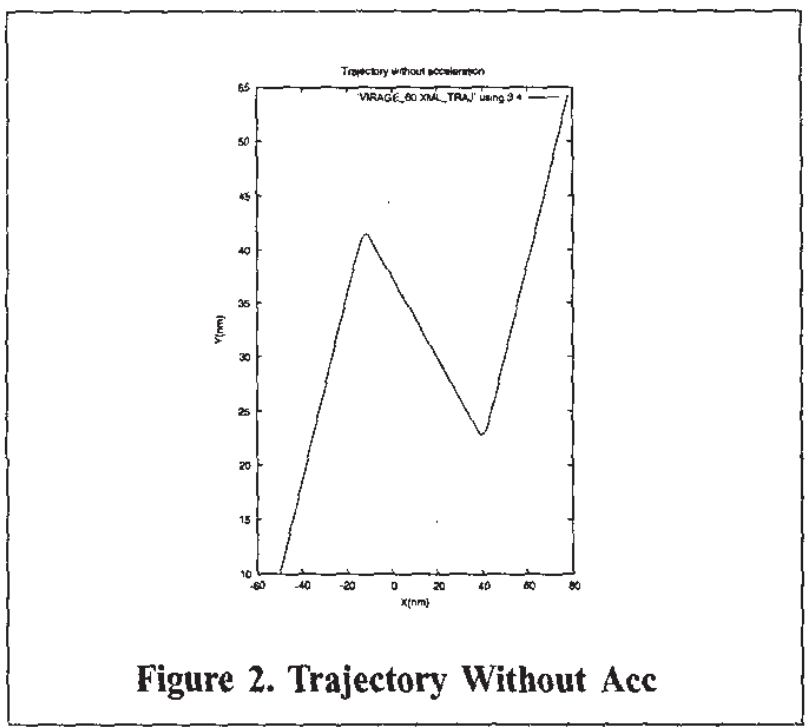

A $30 \mathrm{kts}$ wind coming from the north has also been included in the simulation. After simulation, we have 30 estimated trajectories and one reference trajectory. For each time step, the differences between the estimates and the reference are computed. Wind errors in magnitude $\varepsilon_{\theta}(k)$ and direction $\varepsilon_{\|\underline{w}\|}(k)$ are then computed. The power of those error are evaluated :

$$
\begin{aligned}
R M S_{\varepsilon_{\theta}}(k) & =\frac{1}{N} \sqrt{\sum_{i=1}^{N} \varepsilon_{\theta}^{2}(k i)} \\
R M S_{\varepsilon_{\|\underline{\|}\|}}(k) & =\frac{1}{N} \sqrt{\sum_{i=1}^{N} \varepsilon_{\|\underline{W}\|}^{2}(k i)}
\end{aligned}
$$

The wind estimation is summarized on the figure 3 .

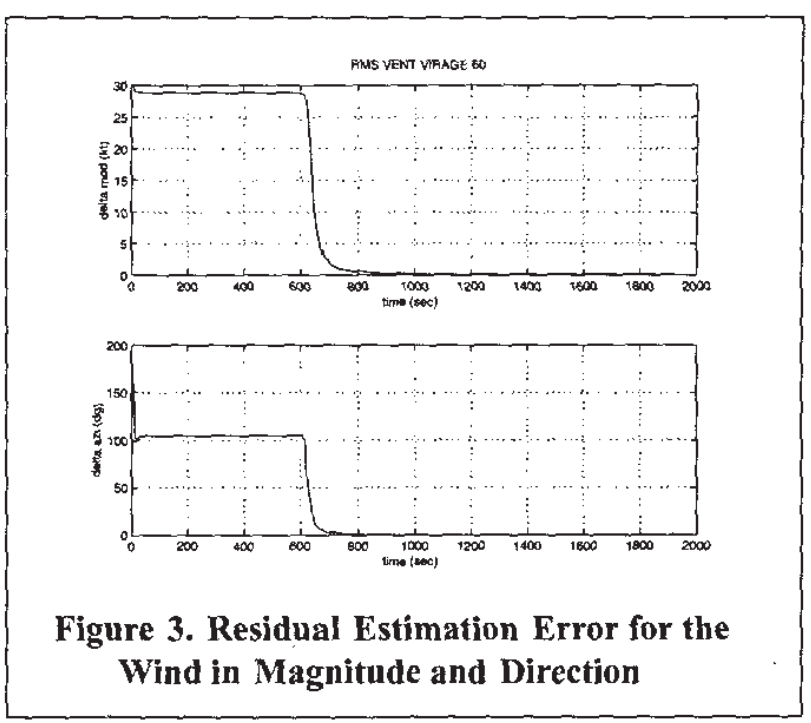

As it can be noticed on the figure, the filter begin to converge to the right wind after the first turn $(600 \mathrm{sec})$. Before, it is not able to estimate the wind because the rebuilt speed may come from the aircraft speed only or from the aircraft speed and the wind. The wind estimation accuracy given by the filter is close to zero showing the model is strongly adapted to the simulation. Unfortunately, this model is only adapted to constant speed trajectory with a full knowledge of the air turning rate. A more realistic framework consist in using trajectories with acceleration and noisy downlinked data such as acceleration rate and air turning rate. This model is able to track any kind of trajectories and to produce the right estimation of the wind. This model with command has been detailed in the previous section*. In order to make run such a filter, information about the noise in the command $\underline{U}(k)$ has to be known to build the covariance matrix $\underline{N}(k)$. The associated standard deviation are the following :

$\sigma_{\gamma_{a}}=0.01 \mathrm{~m} / \mathrm{s}^{2}$ and $\sigma_{\omega_{a}}=0.15^{\circ} / \mathrm{s}$. Furthermore, those noises have been supposed independents, so the matrix $\underline{N}(k)$ is diagonal $\left(\underline{N}(k)[1,1]=\sigma_{\omega_{a}}^{2}\right.$ and $\left.\underline{N}(k)[2,2]=\sigma_{\gamma_{a}}^{2}\right)$. This filter has been tested with a trajectory composed with segments with acceleration period (see figure 4). This trajectory is composed by 9 segments : 5 mn constant speed, $1 \mathrm{mn}$ acceleration $\left(1 \mathrm{~m} / \mathrm{s}^{2}\right), 4 \mathrm{mn}$ constant speed, $1 \mathrm{mn}$ turn $\left(1^{0} / \mathrm{s}\right), 4$ $\mathrm{mn}$ constant speed, $1 \mathrm{mn}$ deceleration, $5 \mathrm{mn}$ constant 
speed, $1 \mathrm{mn}$ acceleration $\left(2 \mathrm{~m} / \mathrm{s}^{2}\right)$ and turn $\left(-1^{0} / \mathrm{s}\right)$ and $10 \mathrm{mn}$ constant speed. A 30 kts north wind has been applied during all trajectory.

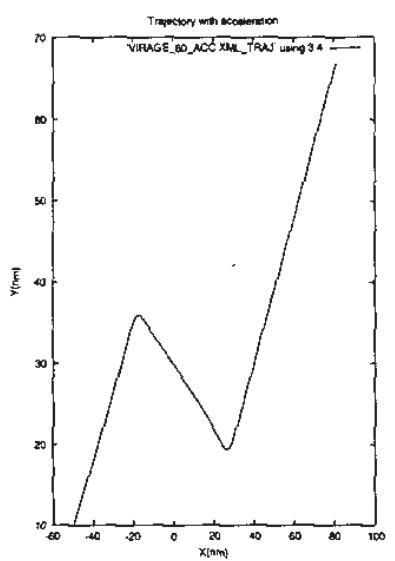

Figure 4. Trajectory With Acc

The residual wind estimation error is given on figure 5. As it can be noticed on this figure the wind estimation is about $0.05 k t s$ for the speed and $0.1^{\circ}$ for the direction.
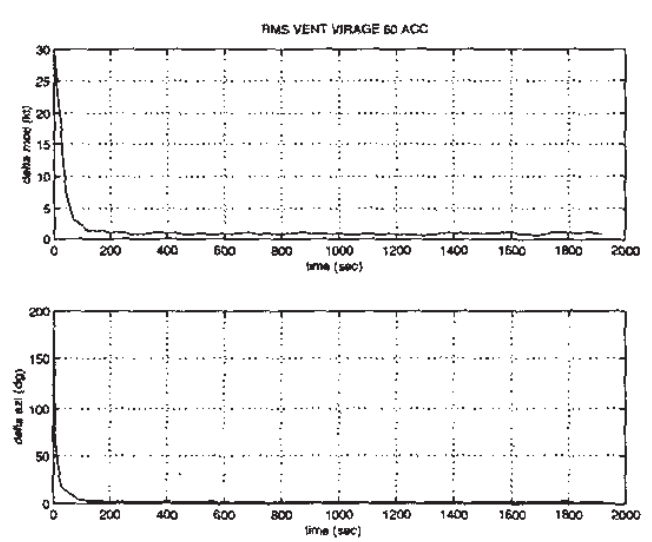

Figure 5. Residual Estimation Error for the Wind in Magnitude and Direction

If the wind is able to change in space (which the real case) the matrix $Q$ has to set up accordingly :

$$
\underline{Q}(k)=\left[\begin{array}{cccccc}
0 & 0 & 0 & 0 & 0 & 0 \\
0 & 0 & 0 & 0 & 0 & 0 \\
0 & 0 & 0 & 0 & 0 & 0 \\
0 & 0 & 0 & 0 & 0 & 0 \\
0 & 0 & 0 & 0 & \left(\sigma_{W}\right)^{2} & 0 \\
0 & 0 & 0 & 0 & 0 & \left(\sigma_{W}\right)^{2}
\end{array}\right]
$$

where $\sigma_{W}$ is the maximum change of averaged wirl speed between two samples and will fix the new accuracy of the filter (a regular value is about $0.5 k t s$ ). This last filter is really adapted for the wind estimation based on the radar tracks. It can address any commercial evolution aircraft and produce accurate wind estimations.

Having now some wind estimations on some points in the airspace (where the aircraft are located), the next step consist in the global wind field interpolation based on the meteorological model called "Shallow-Water". This model is described in [16] and the associated discretization in given in [17].

\section{Vector Spline Interpolation}

\section{Shallow Water Wind Model}

Let $V$ be the vector field giving the wind velocity at each point. We will assume in the following that area of interest is located high enough above the ground level so that viscous effects are negligibles. Assume furthermore that wind field is two dimensional (that is does not depends on altitude). Then the evolution of $V$ is described by the system of equations :

$$
\left\{\begin{array}{l}
\frac{\partial u}{\partial t}+U \frac{\partial u}{\partial x}+\frac{\partial \phi}{\partial x}-f u=0 \\
\frac{\partial v}{\partial t}+U \frac{\partial v}{\partial x}+\frac{\partial \phi}{\partial y}+f v=0 \\
\frac{\partial \phi}{\partial t}+U \frac{\partial v}{\partial x}+\Phi(y)\left[\frac{\partial u}{\partial x}+\frac{\partial y}{\partial y}\right] \frac{\partial \Phi}{\partial y} v=0
\end{array}\right.
$$

with :

$$
V=\left[\begin{array}{c}
U+u \\
v
\end{array}\right]
$$

$U$ the mean west-east component of the wind, $f$ is the coriolis parameter which can be expressed as :

$$
f=2 \Omega \sin \phi_{0}-2 \Omega R^{-1} \cos \phi_{0}
$$

with $\Omega=\frac{2 \pi}{86400}$ the rotation rate of the earth, $\phi_{0}$ the latitude (assumed to be constant in the domain of 
interest) and $R=6.370 \times 10^{6} \mathrm{~m}$ the earth radius. $\Phi$ is the geopotential of the isobaric surface and is written as :

$$
\Phi=\theta(y)+\phi
$$

with :

$$
\theta(y)=\theta_{0}-\left[2 \Omega\left(\sin \phi_{0}\right) y-\Omega R^{-1}\left(\cos \phi_{0}\right) y^{2}\right] U
$$

and :

$$
\phi_{0}=3 \times 10^{4} \mathrm{~ms}^{-2} \quad U=25 \mathrm{~ms}^{-1}
$$

The set of equations 33 is known as shallow water model. With little rewriting, it can be shown that this model has the following synthetic form :

$$
\left(\frac{\partial}{\partial t}+L\right) X=0
$$

with :

$$
X=\left[\begin{array}{l}
u \\
v \\
\phi
\end{array}\right]
$$

and $L$ the differential operator :

$$
L=A \frac{\partial}{\partial X}+B \frac{\partial}{\partial y}+C
$$

where

$$
\begin{aligned}
& A=\left[\begin{array}{ccc}
U & 0 & 0 \\
0 & U & 0 \\
\Phi & 0 & U
\end{array}\right] \quad B=\left[\begin{array}{ccc}
0 & 0 & 0 \\
0 & 0 & 1 \\
0 & \Phi & 0
\end{array}\right] \\
& C=\left[\begin{array}{ccc}
0 & -f & 0 \\
f & 0 & 0 \\
0 & \Phi^{\prime} & 0
\end{array}\right]
\end{aligned}
$$$$
\sigma(y, \xi)=\left[\begin{array}{ccc}
\xi_{1}^{2}\left(\Phi^{2}+U^{2}\right)+f^{2} & \xi_{1} \xi_{2} \Phi+i \xi_{1}\left(\Phi \Phi^{\prime}-2 f U\right) & \left.-i \xi_{1}+\xi_{2} U \Phi\right) \\
\xi_{1} \xi_{2} \Phi-i \xi_{1}\left(\Phi \Phi^{\prime}-2 f U\right. & f^{2}+\xi_{1}^{2} U^{2}+\xi_{2}^{2} \Phi^{2}+\Phi^{\prime 2} & \xi_{1} \xi_{2}(U+\Phi)-i \xi_{2} \Phi^{\prime} \\
i \xi_{1}+\xi_{2} U \Phi & \xi_{1} \xi_{2}(U+\Phi)+i \xi_{2} \Phi^{\prime} & \xi_{1}^{2} U+\xi_{2}^{2}
\end{array}\right]
$$

$L^{t} L$ admits an eigenvector expansion $\left(\phi_{n}\right)_{n \in \mathbb{N}}$ with associated eigenvalues $\left(\lambda_{n}\right)_{n \in \mathbb{N}}$. We will week for a kernel $K(x, y, t, u)$ such that :

$$
\begin{gathered}
\int_{I} \int_{D}\left\langle K(x, y, t, u),\left(-\frac{\partial^{2}}{\partial t^{2}}+L^{t} L\right) X(y, u)\right\rangle d y d u \\
=X(x, t)
\end{gathered}
$$

Formally, we may write :

$$
K(x, y, t, u)=\sum_{n} c_{n}(t, u) \phi_{n}(x) \otimes \phi_{n}(y)
$$

and find the right expression for $c_{n}(t, u)$. In the case $I=\mathbb{R}$ and wind fields of finite energy over time,

\section{Interpolating Vector Splines}

The symbol $\sigma(y, \xi)$ of this differential operator $L$ easily computes as :

$$
\sigma(y, \xi)=\left[\begin{array}{ccc}
-i \xi_{1} U & -f & 0 \\
f & -i \xi_{1} U & -i \xi_{2} \\
-i \xi_{1} \Phi(y) & -i \xi_{2} \Phi(y)+\Phi^{\prime}(y) & -i \xi_{1} U
\end{array}\right]
$$

Since a solution 0 of the Shallow-Water model must fulfill :

$$
\left(\frac{\partial}{\partial t}+L\right) V=0
$$

we will seek for a vector field $V$ such that:

$$
V=\operatorname{argmin} \int_{0}^{T} \int_{D}\left\|\left(\frac{\partial}{\partial t}+L\right) X(x, t)\right\|^{2} d x d t
$$

under the constraints that :

$$
X\left(x_{i}, t_{i}\right)=v_{i}, i=1 \ldots n
$$

if $v_{i}$ is the wind measurement at time $t_{i}$ and location $x_{i}, D$ is the domain of interest and $I$ is the time interval chosen for computation. Assuming that boundary conditions vanish, we obtain that the functional criterion has expression:

$$
\int_{I} \int_{D}\left\langle\left(-\frac{\partial^{2}}{\partial t^{2}}+L^{t} L\right) X(x, t), X(x, t)\right\rangle d x d t
$$

with $L^{t}$ the adjoint operator of operator $L$. Operator $L^{t} L$ has symbol : some computations show that the sought after kernel has expression :

$$
K(x, y, t, u)=\sum_{n} \frac{e^{-\mu_{n}|t-u|}}{2 \mu_{n}} \phi_{n}(x) \otimes \phi_{n}(y)
$$

with : $\mu_{n}=\sqrt{\lambda_{n}}$. The constraints that $X\left(x_{i}, t_{i}\right)=v_{i}, i=1 \ldots n$ may thus be rewritten as :

$$
\int_{I} \int_{D}\left\langle K\left(x_{i}, y, t_{i}, u\right),\left(-\frac{\partial^{2}}{\partial t^{2}}+L^{t} L\right) X(y, u)\right\rangle d y d u=v_{i}
$$

which is much more tractable than the point version. Introducing Lagrange multipliers $\left(\varepsilon_{i}\right)_{i=1 \ldots n}$, the 
problem may be solved and the solution written as :

$$
X(x, t)=\sum_{i=1 \ldots n} \varepsilon_{i} K\left(x_{i}, x, t_{i}, t\right)
$$

so that optimal vector field is a linear combination of kernels evaluated at measurement points $\left(x_{i}, t_{i}\right)_{i=1 \ldots n}$. This is a classical result within the frame of interpolating spline theory, with the restriction that the kernel is not translation invariant. From now, only numeric evaluations of the kernel can be made, based on finding finite elements approximations of the eigenfunctions, then truncating the infinite sum in the kernel expansion at an order where residual is low enough. This yields a piecewise polynomial approximate kernel, which can be computed once and used afterwards (note that if the kernel is computable, finding the wind field expansion is done by solving a linear system).

\section{Conclusion}

This paper has given a new approach for extracting the wind information from the radar tracks. A first linear model has been used to demonstrate that turn observations are enough to estimate the wind around the aircraft. The estimation is only possible if the air turning rate is available at any time. In order to address more realistic trajectories, a second model with downlinked commands has been presented and tested on simulation with acceleration giving good results. This non-linear model has been linearized in order to make run an Extended Kalman Filter. Having wind measures spread in the airspace, the second part of the paper propose an extrapolating method using vector splines. Based on the differential operator of the Sallow-Water model and the observations produced by the Kalman filters, this method build a vector field using kernel functions. The associated eigenfunctions $\left(\phi_{n}\right)$ have to be computed numerically on each point of the grid where the vector field has to be computed. It must be noticed that the Shallow-Water is only valid above $2000 \mathrm{~m}$; this means this method may be used mainly for En-Route traffic.

\section{References}

[1] C. Lefas, "Improved tracking with mode-S data-linked velocity measurements," IEEE Transactions on Aerospace and Electronic Systems, vol. 27, no. 4, pp. 709-714, 1991.

[2] H. Erzberger, et al., "Conflict Detection and Resolution in the Presence of Prediction Error", Proceedings of $1^{\text {th }}$ USA-Europe ATM Seminar. Saclay, FAA-Eurocontrol, June, 1997.

[3] S. Modoloni, "Improving Trajectory Forecasting Through Adaptive Filtering Technique", Proceedings of $5^{\text {th }}$ USA-Europe ATM Seminar. Budapest,

FAA-Eurocontrol, June, 2003

[4] R.E. Cole et al., "Wind Prediction Accuracy for Air Traffic Management Decision Support Tools", Proceedings of $3^{\text {th }}$ USA-Europe ATM Seminar. Napoli, FAA-Eurocontrol, June, 2000.

[5] C.M. Rekkas, C. Lefas and N.J. Krikelis. "Three Dimensional Tracking Using On-Board Measurements", IEEE Transactions on Aerospace and Electronic Systems, vol. 27, no. 4, pp 617-62.4, 1991.

[6] D. Delahaye, "Wind Field Update using Radar Track Data". Master's thesis, Ecole Nationale de l'Aviation Civile, Toulouse, France, June, 1992.

[7] Bradford, "Using Aircraft Radar Tracks to Estimate Winds Aloft", The Lincoln Laboratory Journal, vol. 2, no. 3, 1989.

[8] R.E. Kalman, "A New Approach to Linear Filtering and Prediction Problems", Transactions of the ASME-Journal of Basic Engineering, vol. 82, Series D, pp. 35 45, 1960.

[9] P.S. Maybeck, "Stochastic Models, Estimation, and Control (vol 1 and 2)", Academic Press, 1982.

[10] C.K. Chui and G. Chen, "Kalman Filtering with Real-Time Applications”, Springer-Verlag, 1987.

[11] R.H. Battin, "Astraunotical Guidance", McGraw-Hill, 1984.

[12] R.B. Asher, P.S. Maybeck and R.A.K. Mitchell, "Filtering for precision pointing and tracking with application for aircraft to satellite tracking", Proceedings IEEE Conference Decision and Control, IEEE, Houston, Texas, 1975.

[13] B.D.O. Anderson and J.B. Moore, "Optimal 
Filtering", Prentice Hall, 1979.

[14] G. Welch and G. Bishop, "An Introduction to the Kalman Filter", University of North Carolina. Department of Computer Science, Tech. Rep. TR 95-041, 1995.

[15] S.G. Mohinder, and P.A. Angus, "Kalman Filtering Theory and Practice", Prentice Hall, 1993.
[16] S.E. Cohn, and T.F. Parrish, "The Behavior of the Forcast error Covariances for a Kalman Filter in Two Dimensions", Monthly Weather Review, vol. 119, pp. 1757-1785, 1991.

[17] R.D. Richtmyer, and K.W. Morton, "Difference Methods for Initial-Value Problems", Krieger Publishing Company, 1994. 